\title{
Suicidal ideation and behaviour in human immuno-deficiency virus (HIV) disease
}

\author{
STEPHEN PLATT
}

From the beginning of the Acquired Immune Deficiency Syndrome (AIDS) pandemic a decade ago, it was recognised that psychological disturbance and psychiatric disorder were common features of human immuno-deficiency virus (HIV) disease. Acute psychological symptoms result for the most part from the stresses associated with knowledge of personal infection. Following notification of a positive HIV antibodies test result, the patient is in a state of shock, fearful and anxious about the uncertain prognosis and course of the disease, depressed by the threat the virus poses to feelings of self-esteem and self-control, angry and frustrated by new and involuntary lifestyle restrictions, guilty about past «risky» behaviour and the possible infection of previous partners. While these manifestations of situational distress are extremely common (and reportedly similar in phenomenology, severity and duration to adjustment reactions seen in the context of other major life events or disease diagnoses), a minority of HIV-positive patients may present with a more severe psychiatric illness. This can take the form of a nonorganic (functional) disturbance (mainly adjustment and affective disorders, but also including obsessive-compulsive disorders, substance abuse, schizophreniform and paranoid disorders and personality disorders), or an organic disturbance (such as delirium, dementia, delusional disorder or mood disorder).

Suicidal ideation and behaviour have been reported in the literature in connection with every conceivable aspect of HIV disease and AIDS. Studies of psychiatric manifestations among the «worried well» rev-

Indirizzo per la corrispondenza: Dr. S. Platt, MRC Medical Sociology Unit, 6 Lilybank Gardens, Glasgow G12 8QQ, Scotland, U.K.

Fax $(+44)$ 041-337.23.89 eal a moderate degree of suicide risk in patients diagnosed with AIDS anxiety/panic, AIDS phobia, AIDS delusion and factitious disorder. There have been documented cases of attempts to commit suicide by deliberately acquiring the virus through sexual intercourse with an infected person. «High risk» (but HIVnegative or untested) individuals and individuals who have received an HIV-negative test result have expressed suicidal thoughts or behaved in a self-harmful manner. Turning to those who are actually HIVpositive, we can find evidence of suicidal ideation/behaviour after notification of the test result, after an illness or crisis in HIV disease, after diagnosis of AIDS, and in the terminal phase of AIDS. Some authorities have even gone so far as to claim that thoughts of suicide may be «universal», and actual suicide a «clinical commonplace», among people with HIV disease.

There are certainly prima facie grounds for assuming that this group is likely to be at high risk of suicidal behaviour:

- AIDS is a chronic terminal illness and the rate of suicidal behaviour is higher in such illnesses.

- The suicide rate is higher in medically ill persons, especially where disorders of the central nervous system are involved.

- AIDS is linked with secondary depression and other psychiatric syndromes known to be associated with higher rates of suicidal behaviour.

- Many people with HIV infection have a history of risk-taking behaviour (e.g. injecting drug use, criminal activity).

- There may be biological mechanisms contributing to suicidal behaviour in HIV disease: HIV may exert a direct effect on serotonergic pathways or affect neurotransmitter systems implicated in depression; organic mental disorders that lower communi- 
cation abilities and subsequent coping may impair impulse control and thus increase the likelihood of self-harm.

- There are several psychosocial stressors associated with HIV disease which are likely to increase the risk of suicidal behaviour, including negative societal reaction (stigma, discrimination), loss or withdrawal of social support and long-term dependency and helplessness.

Empirical research findings do not, however, unequivocally point to a strong association between suicidal behaviour and HIV/AIDS. Leaving aside anecdotal evidence and uncontrolled case reports, there are two main tyes of relevant studies: comparative and epidemiological. In the former type, the prevalence of suicidal ideation, plans or behaviour among HIV-positive individuals is compared with that among HIV-negative individuals. Out of five studies which demostrated a reasonable level of methodological rigour, only one found a satistically significant result: people with AIDS (PWAs) were less likely than HIVnegative controls to report lifetime suicidal thoughts or «attempts», with ARC and asymptomatic patients somewhere between these extremes (Orr et al., 1990). The authors speculate that AIDS patients may have underreported suicidal ideation and behaviour due to organicity and psychological processes such as denial and/or preoccupation with physical illness.

Three epidemiological investigations have compared rates of suicide among AIDS patients with rates of suicide in the general population. In the best known study Marzuk et al. (1988) found 12 male AIDS patients aged 20-59 years, resident in New York, who had committed suicide in 1985 . The suicide rate in the AIDS group (680.6 per 100,000 person years at risk) was over 36 times higher than the rate (18.8) in the same age and sex group in the general New York population. Somewhat lower, but still highly significant, relative risks (RRs; i.e. the ratio of suicide rate in AIDS patients to the rate of suicide in the general population) were found in California in 1986 (Kizer et al., 1988; RR =17.0) and in Texas in 1986-87 (Plott et al.,1989, $\mathrm{RR}=16.3$ ).

These findings appear to constitute clear evidence that the risk of suicide is significantly higher among AIDS patients (even if they tell us nothing about the suicide risk associated with asymptomatic HIV infection or ARC, or the parasuicide risk at any stage of HIV disease). Marzuk et al. (1988) believe that the suicide rate in AIDS patients may be an underes- timate due to the effects of ascertainment bias (i.e. the deliberate under-reporting of suicide to avoid stigma and negative socioeconomic consequences; unrecognised suicide as a result of the high "natural» mortality of HIV disease; or misclassification of suicide as undetermined or accident, especially deaths among injecting drug users). However, the RRs may also be misleadingly high because of the failure to compare AIDS suicide rates with suicide rates in more suitable control groups (e.g. gays or injecting drug users without AIDS), while the authors' reporting of rates on a population-time basis and use of RRs have been challenged on technical grounds (Beltangrady \& Glastonbury, 1988).

More importantly, perhaps, we should note that HIV disease-related suicide appears to have had little impact on the overall suicide picture. In the Marzuk et al. (1988) study, the 12 AIDS suicides formed only $3.3 \%$ of all suicides among males aged 20-59 in New York in 1985 while the five AIDS suicides in the Plott et al. (1989) study amount to only $0.2 \%$ of all the suicides in Texas in 1986-87. Furthermore, a detailed «psychological autopsy" investigation of suicide in Finland in 1987-88 failed to uncover a single HIVpositive suicide among 1397 suicides in total (Lonnqvist, 1990); Engleman et al. (1988) found no increase in suicide among white males aged 25-54 in San Francisco over the period 1976-86, and no difference in suicide trends in high risk AIDS census tracts versus low risk AIDS census tracts; and Buehler et al. (1990), noting a reversal of mortlaity trends in young men in the United States since 1983, found less than $0,2 \%$ of the 10248 deaths where the underlying cause included HIV/AIDS could be attributed to suicide, and no difference in suicide trends for states with high versus low incidence of AIDS. "This suggest», they conclude, «that suicides among men with HIV infection has not had a substantial effect on suicide trends».

Beyond that, it is not possible to go very far. The range of studies in the HIV/AIDS/suicidal behaviour field has been disappointingly narrow, methodological deficiences and problems remain widespread and unsovled, and uncorroborated and senstionalist claims abound. Now is the time for researchers to do some calm stocktaking and work towards the development of a feasible and well-directed research programme. The recent WHO Consultation document (WHO, 1990 ) is an important contribution in this endeavour. 


\section{REFERENCES}

Beltangrady M. \& Glastonbury S. (1988). The risk of suicide in persons with AIDS. Journal of the American Medical Association 260, 29.

Buehler J. W., Devine O. J., Berkelman R. L. \& Chevarley F. M. (1990). Impact of the human immunodeficiency virus epidemic on mortality trends in young men, United States. American Journal of Public Health 50, 1080-1086.

Engelman J. E., Hessol N. A., Lifson A. R., Lemp G. F., Mata A., Rutherford G. W., Goldblum P., Bott C. \& Stephens B. (1988). Suicide patterns and AIDS in San Francisco. Paper given at IV International Conference on AIDS, Stockholm.

Kizer K., Green M., Perkins C., Doebbert G. \& Hughes M. (1988). AIDS and suicide in California. Journal of the American Medical Association 260, 1881.
Lonnqvist J. (1990). Suicide and fear of having contracted AIDS. Working paper presented at WHO Consultation on Suicidal Behaviour among AIDS and HIV-infected patients, Bologna, Italy.

Marzuk P., Tierney H., Tardiff K., Gross E., Morgan E., Hsu M. \& Mann J. J. (1988) Increased risk of suicide in people with AIDS. Journal of the American Medical Association 259, 1333-1337.

Orr D., O'Dowd M. A., McKeganey F. P. \& Natali C. (1990). A comparison of self reported suicidal behaviours in different stages of HIV infection. Paper given at VI International Conference on AIDS, San Francisco.

Plott R., Benton S. \& Winslade W. (1989). Suicide of AIDS patients in Texas: a preliminary repcrt. Texas Medicine 85, 40-43.

World Health Organization. Regional Office for Europe (1990). Suicidal Behaviour Among People with HIV and AIDS. Report on a WHO consultation. WHO: Copenhagen. 